\title{
Microtubule Redistribution in Growth Cones Elicited by Focal Inactivation of Kinesin-5
}

\author{
Vidya C. Nadar, Shen Lin, and Peter W. Baas \\ Department of Neurobiology and Anatomy, Drexel University College of Medicine, Philadelphia, Pennsylvania 19129
}

In order for growth cones to turn, microtubules from the central domain must preferentially invade the peripheral domain in the direction of the turn. Recent studies suggest that kinesin-5 (also called Eg5 or kif11) suppresses the invasion of microtubules into the peripheral domain on the side of the growth cone opposite the direction of turning. In theory, kinesin- 5 could elicit these effects by acting on the microtubules within the peripheral domain itself, by acting on microtubules in the central domain, or in the transition zone between these two domains. In rat neurons expressing kinesin-5, we documented the presence of kinesin- 5 in both domains of the growth cone and especially enriched in the transition zone. We then focally inactivated kinesin-5 in various regions of the growth cone, using micro-chromophore-assisted laser inactivation. We found that a greater invasion of microtubules into the peripheral domain occurred when kinesin-5 was inactivated specifically in the transition zone. However, there was no effect on microtubule invasion into the peripheral domain when kinesin- 5 was inactivated in the peripheral domain itself or in the central domain. In other experiments, frog growth cones were observed to turn toward a gradient of a drug that inhibits kinesin-5, confirming that asymmetric inactivation of kinesin- 5 can cause the growth cone to turn. Finally, expression of a phospho-mutant of kinesin-5 resulted in greater microtubule invasion throughout the peripheral domain and an inhibition of growth cone turning, implicating phosphorylation as a means by which kinesin-5 is regulated in the growth cone.

\section{Introduction}

The turning of the axon toward relevant signaling cues involves a change in the distribution of microtubules at its distal tip, termed the growth cone (Conde and Caceres, 2009; Geraldo and Gordon-Weeks, 2009; Lowery and Van Vactor, 2009; Dent et al., 2011). Growth cones consist of two distinct domains called the central domain and the peripheral domain, the former of which is the microtubule-rich region contiguous with the axonal shaft and the latter of which is the actin-rich lamellar region that includes filopodia (Goldberg and Burmeister, 1986; Aletta and Greene, 1988). For the axon to turn, microtubules from the central domain must invade the peripheral domain in a polarized fashion in the direction of the turn (Tanaka and Kirschner, 1995). Several studies have shown that the invasion into filopodia of microtubules is dependent upon their dynamic properties (Williamson et al., 1996; Challacombe et al., 1997; Buck and Zheng, 2002; Suter et al., 2004), but these studies do not reveal how microtubules preferentially invade the peripheral domain on one side of the growth cone versus the other.

\footnotetext{
Received Jan. 11, 2012; revised Feb. 13, 2012; accepted March 2, 2012.

Author contributions: V.N. and P.W.B. designed research; V.N. and S.L. performed research; V.N. and P.W.B. analyzed data; V.N. and P.W.B. wrote the paper.

This work was funded by grants from the National Institutes of Health and the National Science Foundation to P.W.B. Shen Lin was supported by a Postdoctoral Fellowship from the Craig H. Neilsen Foundation. We thank Drs. Mei Liu and Wenqian Yu of our laboratory for advice and assistance. We thank Dr. James Zheng of Emory University for instruction and advice on the drug gradient experiments.

The authors declare no competing financial interests.

Correspondence should be addressed to Peter W. Baas, Ph.D., Department of Neurobiology and Anatomy, Drexel University College of Medicine, 2900 Queen Lane, Philadelphia, PA 19129. E-mail: pbaas@drexelmed.edu.

DOI:10.1523/JNEUROSCI.0144-12.2012

Copyright $\odot 2012$ the authors $\quad 0270-6474 / 12 / 325783-12 \$ 15.00 / 0$
}

Recent studies suggest critically important roles for molecular motor proteins in regulating microtubule distribution in growth cones. The first of these studies demonstrated that cytoplasmic dynein, a minus end-directed motor protein, is required for a portion of the microtubules to overcome the retrograde flow of filamentous actin within the growth cone and thereby enter the peripheral domain (Myers et al., 2006; Grabham et al., 2007). Subsequently, it was shown that the homotetrameric motor protein called kinesin-5 (also called Eg5 or kif11), best known for its essential role in mitosis (Sawin et al., 1992; Ferenz et al., 2010), is required for growth cones to turn in response to signaling cues (Nadar et al., 2008). Inhibition of kinesin-5 results in nonpolarized invasion of microtubules throughout the peripheral domain, causing the growth cones to ignore these cues (Nadar et al., 2008). Kinesin-5 appears to function, at least in part, by generating forces that oppose the forces generated by cytoplasmic dynein, thereby resisting microtubule entry into the peripheral domain. The question now arises as to where kinesin- 5 acts in the growth cone and how its forces are polarized to impose directionality on growth cone turning.

Based on localization studies, we have proposed that kinesin-5 acts mainly in the transition zone between the central and peripheral domains to regulate the entry of microtubules from the former into the latter (Nadar et al., 2008). In the present study, we first analyzed the behavior of kinesin-5-EGFP in growth cones using live-cell imaging, and then investigated where in the growth cone kinesin- 5 functions by focally inactivating kinesin- 5 in different regions of the growth cone using micro-chromophoreassisted laser inactivation (CALI). In addition, we determined whether growth cones turn toward a gradient of monastrol, a 
potent and specific anti-kinesin-5 drug, and we explored the merits of a scenario whereby phosphorylation of kinesin-5 is a physiological means by which its activity is regulated in growth cones.

\section{Materials and Methods}

Rat kinesin-5-EGFP constructs. The full-length rat kinesin-5 cDNA sequence (GenBank No.XM_001060913) was obtained by High Fidelity $\mathrm{PCR}$, using rat fetal brain cDNA as a template. The restricted DNA was cloned into pEGFP-N1 (Clontech) for expression in eukaryotic cells. Site-directed mutagenesis was conducted to generate a construct bearing a mutation in which the threonine in the so-called bimC box of the tail domain was changed to alanine. This mutant, termed T926A, has been shown in previous studies to negatively impact the ability of kinesin-5 to function, presumably by suppressing its capacity to interact with microtubules (Blangy et al., 1995; Sawin and Mitchison, 1995; Cahu et al., 2008).

Cell culture and transfection. Time-pregnant Sprague Dawley rats were obtained from Charles River and the postnatal pups (P0-P1), including both males and females, were used to obtain primary neurons from the sympathetic ganglia. The neurons were then dissociated as described previously (He et al., 2005). The dissociated cells were transfected (using the Amaxa nucleofector) with $15 \mu \mathrm{g}$ of kinesin-5-EGFP and $15 \mu \mathrm{g}$ of mCherry-EB3 for coimaging and micro-CALI experiments. EB3 appears, when expressed as a fluorescent fusion protein, as fluorescent comets at the plus ends of microtubules as they undergo assembly (Stepanova et al., 2003; Nadar et al., 2008). For other experiments, the neurons were transfected with either wild-type kinesin-5-EGFP or T926A kinesin-5-EGFP. The transfected cells were plated on $0.1 \mathrm{mg} / \mathrm{ml}$ poly-D-lysine-coated plastic dishes in L-15 medium. After $24 \mathrm{~h}$, the cells were replated onto 0.1 $\mathrm{mg} / \mathrm{ml}$ poly-D-lysine-coated glass coverslips at a density of 7500 cells per dish and were imaged after $12-16 \mathrm{~h}$, by which time the axons displayed robust growth cones at their tips. Rat neurons were used for all experiments except for the drug gradient experiments, in which we used frog neurons (see below).

Live-cell coimaging of neurons expressing both kinesin-5-EGFP and $m$ Cherry-EB3. Growth cones of neurons that had been successfully cotransfected with the two constructs were identified, and sequential images from the green and red channels were obtained every second using the multidimensional acquisition module of the AxioVision software. The images were then analyzed for kinesin- 5 distribution, relative to the different regions of the growth cone and relative to the positions of the EB3 comets.

Micro-CALI. Kinesin-5 was focally inactivated in different regions of the growth cone using micro-CALI. This involved irradiation of growth cones expressing kinesin-5-EGFP with a laser at the wavelength of 488 $\mathrm{nm}$. The laser irradiation of EGFP molecules results in the release of free oxygen radicals, which inhibits the function of the protein fused to the EGFP in the irradiated region (McLean et al., 2009). Time-lapse images of mCherry-EB3 in cotransfected neurons were obtained every $3 \mathrm{~s}$ for 1 $\mathrm{min}$. This was followed by irradiation at $488 \mathrm{~nm}$ (MicroPoint laser; Andor Technology) in different regions of the growth cone by laser pulses for $10 \mathrm{~s}$. "Mosaic" software was used to draw the outline of the region to be irradiated. Immediately after the laser irradiation, time-lapse images were obtained over a period of $1 \mathrm{~min}$ with images captured every $3 \mathrm{~s}$. The data used for quantification in Figures 5 and 6 were obtained from timelapse images obtained for $1 \mathrm{~min}$ before and after laser irradiation at $3 \mathrm{~s}$ intervals. Time-lapse movies were analyzed by counting the number of EB3 comets that entered the peripheral domain adjacent to the region of irradiation during the $1 \mathrm{~min}$ before and after the irradiation. The number of comets per second was calculated and a ratio of number of comets after and before the irradiation was obtained.

Drug gradient studies. Dissociated cells were obtained from the neural tube of Xenopus frog embryos (stage 19-22) and then plated on polylysine/laminin-coated coverslips as described previously (Gomez et al., 2003; Guirland et al., 2003). Imaging was performed 6-8 h after plating, by which time the axons displayed prominent growth cones at their tips. A local gradient of monastrol (or dimethylsulfoxide [DMSO] vehicle as a control) was created by the pipette application method as described previously (Guirland et al., 2003); $10 \mu \mathrm{M}$ of monastrol (1000× the concentration used in bath application experiments) was used in the glass pipette. The tip of the pipette was positioned $50 \mu \mathrm{m}$ away from the center of the growth cone at an angle of $45^{\circ}$ from the initial direction of extension. Images were obtained every $5 \mathrm{~min}$ for a maximum period of 30 $\mathrm{min}$. The position of the pipette tip was adjusted every $10 \mathrm{~min}$ so that the distance between the tip and the growth cone remained constant. The growth cones were analyzed for turning behavior by drawing a central axis line through the center of the growth cone and the axonal shaft. The growth cones that moved away from the axis were counted as "turned," and were distinguished as to whether they turned toward the pipette tip or away from it. If the axonal axis remained parallel to the drawn line, it was scored as "no turn." The turning angle was determined as described previously (Buck and Zheng, 2002), and the average turning angle was plotted for DMSO (control) and monastrol-treated groups. These data were also represented as a cumulative distribution. For the growth cones that did not turn, we determined the growth cone area on either side of the central axis.

Studies with the T926A kinesin-5-EGFP mutant. Studies were conducted to compare features of axons and growth cones of neurons expressing moderate levels of wild-type kinesin-5-EGFP with neurons expressing similar levels of the T926A kinesin-5-EGFP phospho-mutant. Axonal length was assessed as in previous studies (Myers and Baas, 2007). Images of mCherry-EB3 in growth cones of neurons cotransfected with the kinesin- 5 constructs and mCherry-EB3 were obtained every second for $3 \mathrm{~min}$ at an exposure time of $300 \mathrm{~ms}$ and $1 \times 1$ binning using the AxioVision software. The number of EB3 comets was quantified as described previously (Nadar et al., 2008). To assay growth cone turning, we used the "scratch assay" as in previous studies (Myers et al., 2006). For this, neurons were transfected with kinesin-5-EGFP or T926A kinesin-5EGFP and plated on $0.1 \mathrm{mg} / \mathrm{ml}$ poly-D-lysine-coated dishes. The cells were replated onto coverslips with alternating laminin and laminin-free stripes and fixed after $4-5 \mathrm{~h}$, by which time many of the axons had reached the border. The pattern was created starting with a surface completely covered with laminin, and then scratching away thin regions of the laminin using a metallic needle to generate alternating stripes where laminin was present and stripes where it was not (Myers et al., 2006). All transfected neurons that were not in contact with the laminin/nonlaminin border were used for analyses. The growth cones of axons that were parallel to the border throughout their entire length were excluded. These cultures were triple-labeled with rabbit anti-GFP, mouse anti- $\beta 3$ tubulin (neuron-specific tubulin), and rabbit anti-laminin. Growth cones of transfected neurons that exhibited turning were counted and the percentage of growth cones that crossed the border was determined. An axon was scored as having turned if the angle between its long axis and the laminin-free region was $<45^{\circ}$, as in earlier work using this assay (Myers et al., 2006).

\section{Results}

\section{Visualization of kinesin-5 in growth cones}

Our plan for the micro-CALI studies was to coexpress EGFPkinesin-5 and mCherry-EB3 in rat sympathetic neurons, so that we could focally inactivate the former in various regions of the growth cone and then visualize the impact on microtubule distribution with the latter. EB3 is a microtubule end-binding protein that, when expressed as a fluorescent fusion protein, appears as fluorescent comets at the plus ends of microtubules as they undergo bouts of assembly (Stepanova et al., 2003; Nadar et al., 2008). In preparation for those studies, we first visualized the two constructs, to ascertain the distribution of EGFP-kinesin-5 in the growth cone as well as its distribution relative to the microtubule array. To focally inactivate EGFP-kinesin-5 in a particular region of the growth cone, we first needed to know if it was present in that region.

As expected, neurons expressing high levels of kinesin-5EGFP displayed an overexpression phenotype of shorter, less 

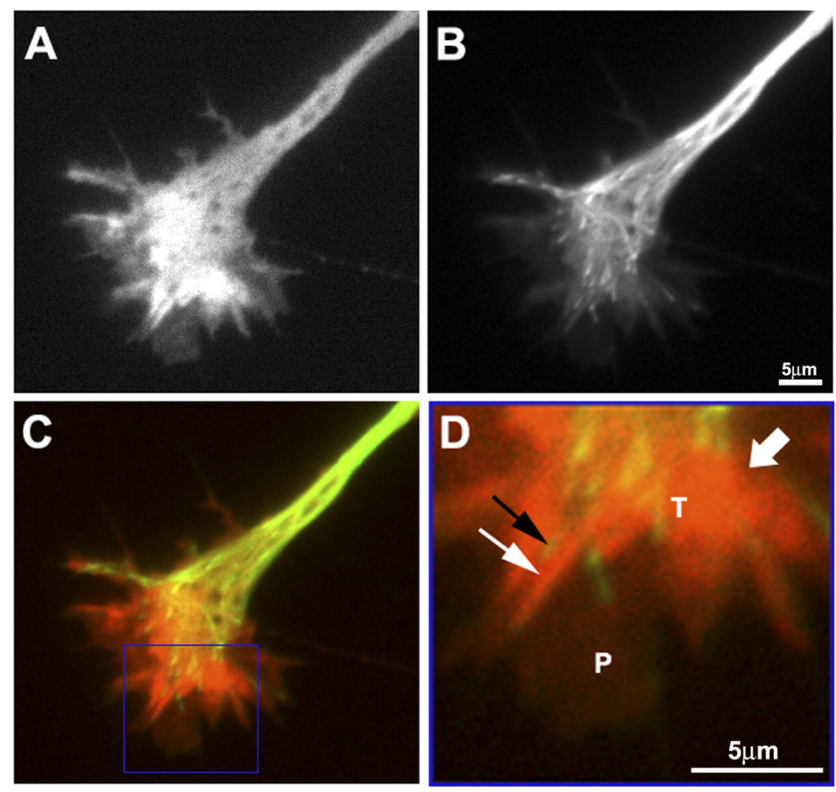

Figure 1. Localization of kinesin-5-EGFP. A, Growth cone of neurons expressing kinesin-5EGFP showing enrichment of kinesin-5 in the transition zone. $\boldsymbol{B}$, The same growth cone transfected with $m$ Cherry-EB3 showing the distribution of microtubule plus ends. Scale bar, $5 \mu \mathrm{m}$. $C$, Overlay of $\boldsymbol{A}$ and $\boldsymbol{B}$ where kinesin-5-EGFP is shown in red and $\mathrm{mCherry}-\mathrm{EB} 3$ is shown in green. (The clarity was better in the overlay if we switched the colors). $\boldsymbol{D}$, Enlarged image of area of the growth cone in blue box in C showing kinesin-5 in a microtubule-shaped blur in the transition zone (thinner white arrow), with an EB3 comet appearing just behind it (black arrow). The thicker white arrow denotes the enrichment of kinesin- 5 in the transition zone (T). Peripheral domain is indicated by $P$.

branched axons with small growth cones (Myers and Baas, 2007). We chose for our analyses moderate expressers that displayed morphologies indistinguishable from nonexpressers. In these neurons, there was diffuse fluorescence throughout the growth cone, but notably stronger fluorescence in the transition zone (Fig. 1A) compared with the peripheral or central domains (as well as the axonal shaft). This was reminiscent of our previous immunofluorescence observations on kinesin-5 distribution in these neurons (Nadar et al., 2008). Notably, the transition zone was not richer in EB3 fluorescence than the central domain or the axonal shaft (Fig. $1 B$ ), but the kinesin-5 fluorescence was notably more intense. This is consistent with the previous immunofluorescence results indicating a true enrichment of kinesin-5 in the transition zone. In the transition zone, a portion of the kinesin5-EGFP fluorescence was observed to form streak-like blurs that roughly/partially colocalized with the invading microtubules, as visualized with the mCherry-EB3 (Fig. 1C,D). Based on the current observations, we conclude that kinesin-5-EGFP is indeed present in both domains of the growth cone and in the transition zone, and hence that kinesin-5 could theoretically function in any or all of these locations.

One more issue relevant to our plans for the micro-CALI studies was whether we could use neurons expressing EGFP alone as the control. We were initially concerned that the higher levels of kinesin-5-EGFP in the transition zone might result in a greater adsorption of energy in this region relative to other regions and hence might produce a nonspecific disruptive effect that might permit microtubules to advance, regardless of the actual function of kinesin-5. To control for this possibility, EGFP in the controls would have to be present in at least the same levels in control growth cones as the kinesin-5-EGFP in comparable regions of the experimental growth cones. We found that this was indeed the case, as illustrated by side-by-side images displayed in quantitative pseudocolor. In such images, the levels of fluorescence are clearly higher in all regions of the growth cones of EGFPexpressing neurons (Fig. $2 A$ ) than in any region of the growth cone of the EGFP-kinesin-5-expressing neurons (Fig. $2 B$ ). This includes the transition zone, where the fluorescence is highest. Thus, we felt it was reasonable to use neurons expressing EGFP (together with mCherry-EB3) as the control for our micro-CALI studies.

\section{Focal inactivation of kinesin-5 in growth cones}

In previous studies from other laboratories using micro-CALI to focally inactivate cytoskeletal proteins in growth cones, malachite green-conjugated antibodies against the target protein were introduced into the cells and specific regions of the growth cones were irradiated (Liu et al., 1999; Mack et al., 2000; Koester et al., 2007). The contemporary approach for micro-CALI is to express a GFP-fusion for the target protein and irradiate specific regions of the cell with a laser at a wavelength of $488 \mathrm{~nm}$. For most proteins, it would presumably be necessary to at least partially deplete the endogenous protein while expressing the GFP-fusion, so that the laser does not merely inactivate the expressed protein, leaving the endogenous protein functional. For our studies, we have the advantage that kinesin-5 forms homotetramers (Cole et al., 1994; Kashina et al., 1996) so that the GFP-fused protein should intermingle with the endogenous protein to generate tetramers containing both. Hence, it should not be necessary to deplete the endogenous kinesin-5 for our micro-CALI experiments, as long as the expression is not so high as to elicit an overexpression phenotype (Myers and Baas, 2007).

Using neurons expressing mCherry-EB3 and either kinesin-5EGFP or EGFP alone as a control, we irradiated the three different regions of the growth cone discussed earlier, namely the central domain, the peripheral domain, and the transition zone, and then acquired time-lapse images of the EB3 comets before and after laser irradiation. The number of EB3 comets in the peripheral domain after laser irradiation was compared with the number before laser irradiation. If our hypothesis is correct that kinesin-5 functions specifically in the transition zone of the growth cones, we would expect to see increased microtubule invasion into the peripheral domain after laser irradiation in the case of neurons expressing kinesin-5-EGFP and irradiated in the transition zone (Fig. 3A4), but no significant effect on the number of microtubules invading the peripheral domain in the five other experimental groups (Fig. $3 A 1-A 3, A 5, A 6$ ).

It is worth noting that EGFP also serves as a volume marker, as it generally fills the cytoplasm evenly. As can be seen in Figure 2, the kinesin-5-EGFP shows the highest levels in the area beyond the palm of the growth cone contiguous with the axonal shaft, which is the vicinity of the transition zone. In contrast, the EGFP (volume marker) control shows the highest levels in that palm region, with levels tapering off in the more outer regions of the central domain, and especially in the transition zone and peripheral domains. These observations provide further confidence that the kinesin-5-EGFP shows a true enrichment in the transition zone.

The domain structure of the growth cone has been established in previous work on the basis of the distribution of microtubules and actin filaments, with the transition zone defined as the region between the microtubule-rich central domain and the actin-rich peripheral domain (Medeiros et al., 2006; Geraldo and GordonWeeks, 2009). It was crucial for our micro-CALI studies that we chose relatively broad and spread out growth cones in which the 
different domains could be distinguished by morphology and by the behavior and distribution of the EB3 comets. The region of the growth cone where the fluorescence produced by the mCherry-EB3 was brightest, with large numbers of comets often moving in nonlinear trajectories, was considered to be the central domain, whereas the region containing sparse more linear comets was considered to be the peripheral domain. The area in between where the comets often originated and sometimes moved laterally was considered to be the transition zone. These determinations were made before laser irradiation, so as to prevent any potential bias in our interpretation of the results. Figure $3 B-D$ is an example of a growth cone of a neuron expressing kinesin-5-EGFP and irradiated in the peripheral domain. Consistent with our prediction for this experimental scenario, the comparison of the time-lapse image of EB3 comets obtained before laser irradiation (Fig. $3 B$ ) with the time-lapse image after laser irradiation (Fig. 3D) did not reveal a significant change in the number of comets entering the peripheral domain in response to laser irradiation. Quantification of all growth cones from neurons expressing kinesin-5-EGFP and irradiated in the peripheral domain $(n=17)$ indicated that $53 \%$ of the growth cones exhibited an increase in the number of EB3 comets entering the peripheral domain, whereas $47 \%$ of the growth cones exhibited a decrease. Similar results were obtained when growth cones expressing kinesin-5-EGFP were laser irradiated in the central domain $(n=25)$ where $44 \%$ of the growth cones showed an increase; $44 \%$ also showed a decrease, whereas $12 \%$ of the growth cones did not show a change.

In the case of control neurons transfected with EGFP and irradiated in the transition zone (Fig. $4 A-C$ ), only $41 \%$ of the growth cones showed an increase whereas $36 \%$ of the growth cones showed a decrease and $23 \%$ of the growth cones showed no difference. Growth cones were also laser irradiated in the central domain and the peripheral domain (Fig. 4D-I). Quantification of the number of EB3 comets in the peripheral domain of growth cones irradiated in the peripheral domain $(n=23)$ revealed that $57 \%$ of the growth cones showed an increase, $39 \%$ of the growth cones showed a decrease, and $4 \%$ of the growth cones did not show a change. Similarly, in growth cones of neurons transfected with EGFP and irradiated in the central domain $(n=15), 40 \%$ showed an increase, $53 \%$ showed a decrease, and $7 \%$ showed no change in the number of EB3 comets invading the peripheral domain. shown in Figure 1. Bar, $5 \mu \mathrm{m}$. domain. Scale bar, $5 \mu \mathrm{m}$.

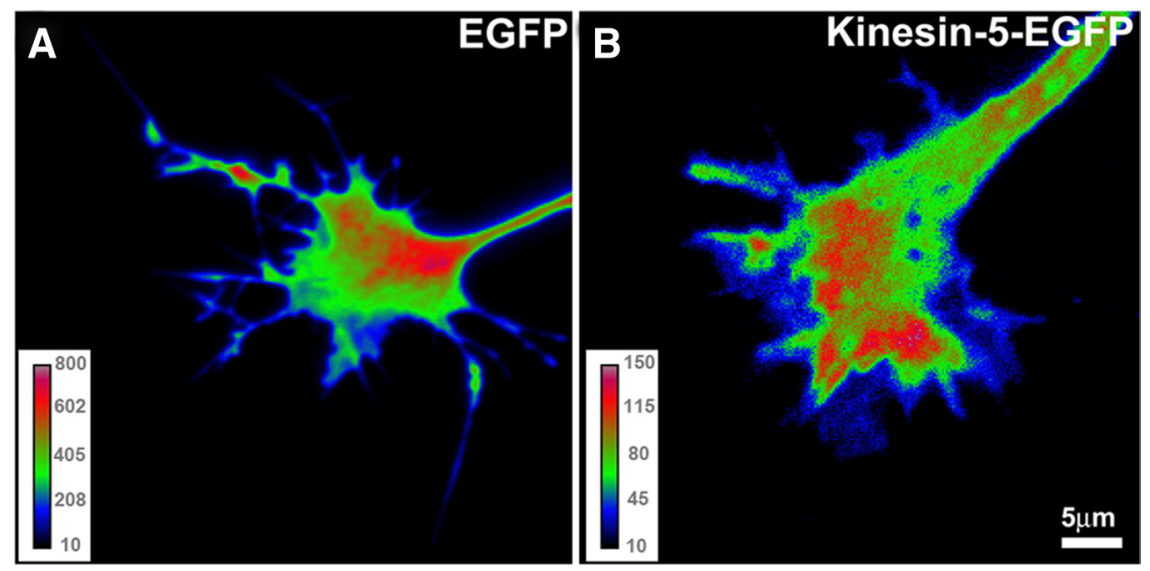

Figure 2. Quantitative pseudocolored images of growth cones of neurons expressing EGFP or kinesin-5-EGFP. $\boldsymbol{A}$, Pseudocolored image of growth cone of neuron expressing EGFP. The scale is in the range of 10 to 800 . $\boldsymbol{B}$, Pseudocolored image of growth cone of neuron expressing kinesin-5-EGFP. The scale is in the range of 10 to 150 . The growth cone shown in $\boldsymbol{B}$ is the same as the growth cone
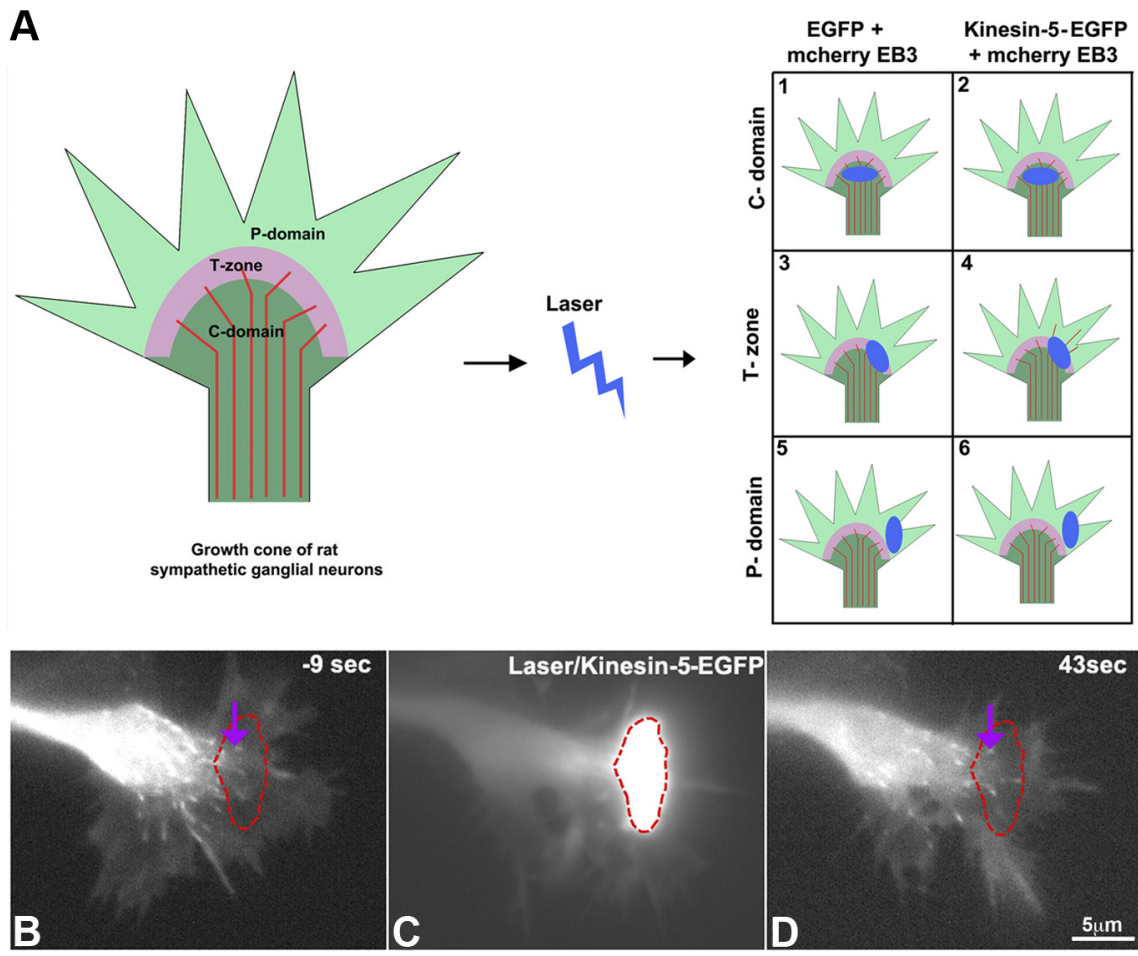

Figure 3. Experimental paradigm for micro-CALI. $\boldsymbol{A}$, Growth cones of neurons transfected to express mCherry-EB3 and either kinesin-5-EGFP or EGFP were laser irradiated in the central domain, peripheral domain, or transition zone as indicated by the 6 different schematic growth cones. The blue region indicates the area of irradiation; microtubules are shown in red. Each schematic shows our predicted outcome based on our hypothesis that the forces generated by kinesin- 5 are most relevant in the transition zone. $\boldsymbol{B}-\boldsymbol{D}$, Fluorescence images of a growth cone of a neuron transfected with mCherry-EB3 and kinesin-5-EGFP. $\boldsymbol{B}$, Image taken 9 s before irradiation in the peripheral domain. $C$, Image taken during irradiation. $\boldsymbol{D}$, Image taken $43 \mathrm{~s}$ after irradiation. Red outline indicates the area of irradiation; the purple arrow denotes the EB3 comets entering the peripheral domain. There is no apparent change in the degree to which microtubules invade the peripheral domain as a result of kinesin- 5 inactivation in the peripheral

Thus, irradiation in the central domain or peripheral domain of growth cones of neurons expressing either kinesin-5-EGFP or EGFP did not have a significant effect on the number of EB3 comets entering the peripheral domain. The same was true of neurons expressing EGFP and irradiated in the transition zone.

Next, we irradiated growth cones in the transition zone of neurons expressing kinesin-5-EGFP. Time-lapse images of the 

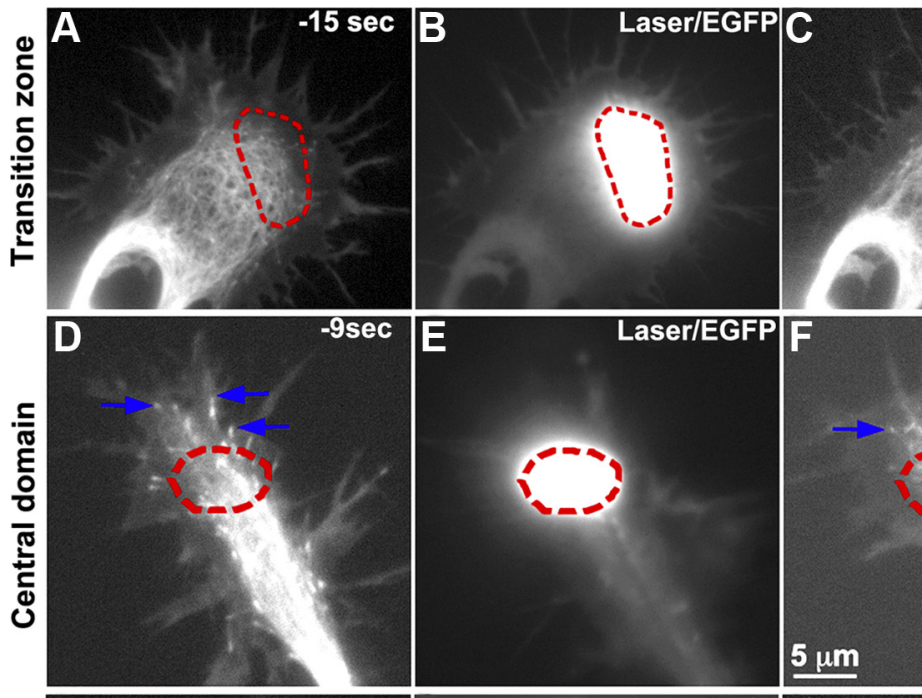

Laser/EGFP/F
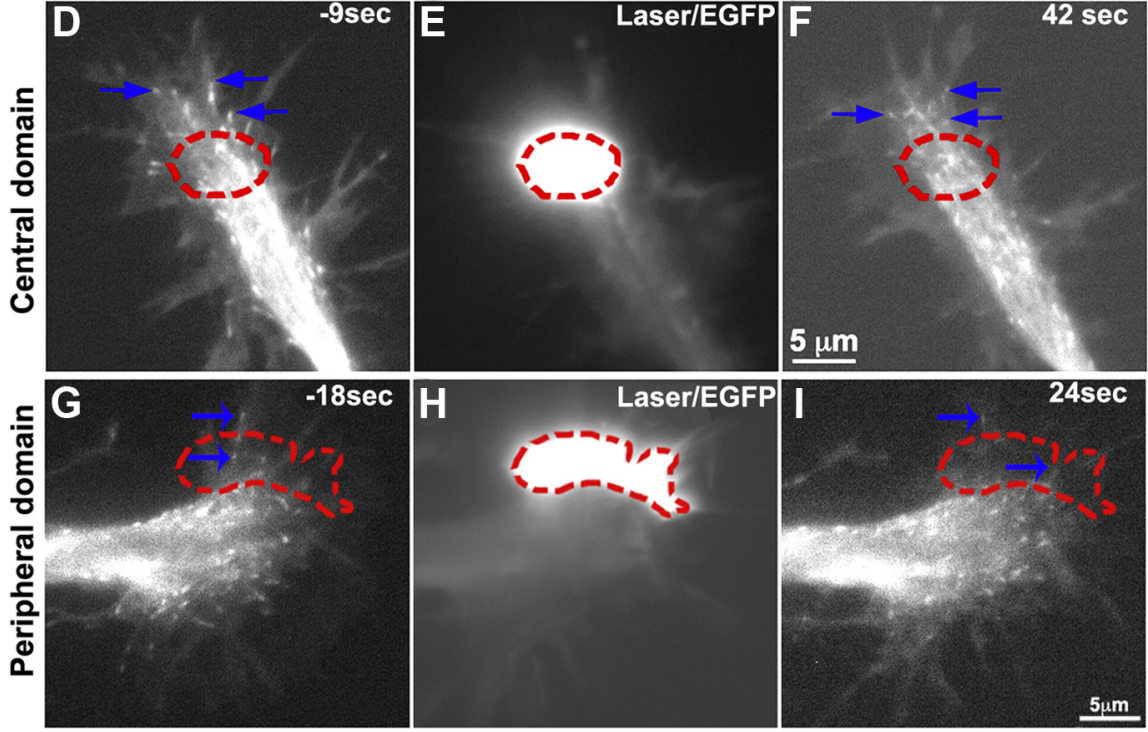

Figure 4. Time-lapse images of growth cones of neurons expressing EGFP and irradiated in different regions. $A-C$, Fluorescence images of a growth cone irradiated in the transition zone; the red outline indicates the region of irradiation. $\boldsymbol{A}$, Image taken $15 \mathrm{~s}$ before irradiation. $\boldsymbol{B}$, Image taken during irradiation. $\boldsymbol{C}$, Image taken $60 \mathrm{~s}$ after irradiation. $\boldsymbol{D}, \boldsymbol{E}$, Fluorescence images of a growth cone irradiated in the central domain; the red outline indicates the region of irradiation and the blue arrows indicate EB3 comets in the peripheral domain. $\boldsymbol{D}$, Image taken $9 \mathrm{~s}$ before irradiation. $\boldsymbol{E}$, Image taken during irradiation. $\boldsymbol{F}$, Image taken $42 \mathrm{~s}$ after irradiation. $\mathbf{G}-\boldsymbol{I}$, Fluorescence images of a growth cone irradiated in the peripheral domain; the red outline indicates the region of irradiation and the blue arrows indicate EB3 comets in the peripheral domain. $\boldsymbol{G}$, Image taken $18 \mathrm{~s}$ before irradiation. $\boldsymbol{H}$, Image taken during irradiation. I, Image taken $24 \mathrm{~s}$ after irradiation. Scale bars, $5 \mu \mathrm{m}$.

growth cone taken after laser irradiation (Fig. $5 F-J$ ) show an increased number of EB3 comets in the peripheral domain compared with before the laser irradiation (Fig. $5 A-D$ ). This is clearer in higher magnification images (Fig. $5 K, L$ ). When growth cones of neurons expressing EGFP alone were irradiated in the transition zone, the number of EB3 comets in the peripheral domain did not change after laser irradiation. The quantification of the number of the EB3 comets that entered the peripheral domain in response to the laser irradiation shows that, in contrast to growth cones irradiated in the central domain and peripheral domain, all $(n=28)$ of the growth cones irradiated in the transition zone showed an increase in the number of EB3 comets in the peripheral domain. Almost $61 \%$ of the growth cones exhibited $>2$-fold increase in the ratio of invasion of EB3 comets in response to the micro-CALI.

To quantify the changes in response to laser irradiation, we plotted the ratio of the number of comets invading the peripheral domain after and before irradiation for each of the growth cones that were irradiated in the central domain, peripheral domain, or the transition zone. The data used for this quantification were obtained from all the images acquired every $3 \mathrm{~s}$ for a $1 \mathrm{~min}$ period before and after laser irradiation. Based on our hypothesis as depicted schematically in Figure $3 A$, we expected to see an increase in microtubule invasion only in growth cones of neurons expressing kinesin-5-EGFP and irradiated in the transition zone. The quantifications of the six groups with the adjacent schematic

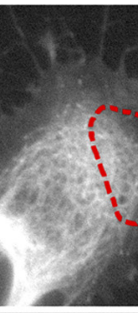

$60 \sec$

depicting the region of irradiation are shown in Figure 6. In addition to some of the growth cones not showing any change in the number of EB3 comets invading the peripheral domain after laser irradiation, the growth cones in all of the groups (except for the growth cone of kinesin-5EGFP neurons irradiated in the transition zone) sometimes showed an increase and sometimes showed a decrease in the number of the EB3 comets invading the peripheral domain. This is to be expected, based on the dynamic nature of the microtubule array within living growth cones. Notably, all of the growth cones of neurons expressing kinesin-5-EGFP and irradiated in the transition zone showed an increase in the number of EB3 comets invading the peripheral domain. Also, the amount of increase in this group in the different growth cones is much higher than that seen in any of the other five groups.

Comparison of the average of the ratios of microtubule invasion in growth cones of neurons expressing EGFP and kinesin-5-EGFP, respectively (the data from Fig. 6 were used here) and irradiated in the central domain $(0.93 \pm 0.09 ; 1.01 \pm$ $0.06)$, peripheral domain (1.03 \pm 0.06 ; $0.98 \pm 0.07)$, and transition zone $(1.1 \pm$ $0.1 ; 3.38 \pm 0.4)$ indicates a significant increase in the ratio only in growth cones irradiated in the transition zone of neurons expressing kinesin-5-EGFP (Fig. 7) $p \leq 0.00001$ (two-tailed $t$ test). In summary, inactivation of kinesin-5 in the transition zone (but not in the peripheral or central domains) results in a clear and consistent increase in microtubule invasion into the peripheral domain, similar to the effect observed in growth cones of neurons globally depleted of kinesin-5.

\section{Growth cones turn toward a gradient of monastrol}

The limitation of the micro-CALI approach is that we can only focally ablate kinesin-5 with confidence for a period of several seconds. While this is adequate to document alterations in microtubule distribution, it is not a sufficient period of time to assay for the turning of the growth cone in response to the changes in microtubule distribution. Given that the growth cone is normally a highly protrusive structure, we found that we could not even use subtle changes in protrusive activity as an indirect indicator of the propensity of the growth cone to turn in response to the focal inactivation. Therefore, we sought another method to determine whether we could induce axons to turn by experimentally inactivating kinesin-5 in an asymmetric fashion in the growth cone. For this, we used a drug gradient approach that has proven successful in previous studies on the cytoskeletal mechanisms of growth cone turning.

In an earlier study of this kind, growth cones were observed to turn toward a gradient of taxol (a microtubule-stabilizing drug) but away from a gradient of nocodazole (a microtubuledepolymerizing drug), thus demonstrating the importance of microtubule dynamics in growth cone turning (Buck and Zheng, 

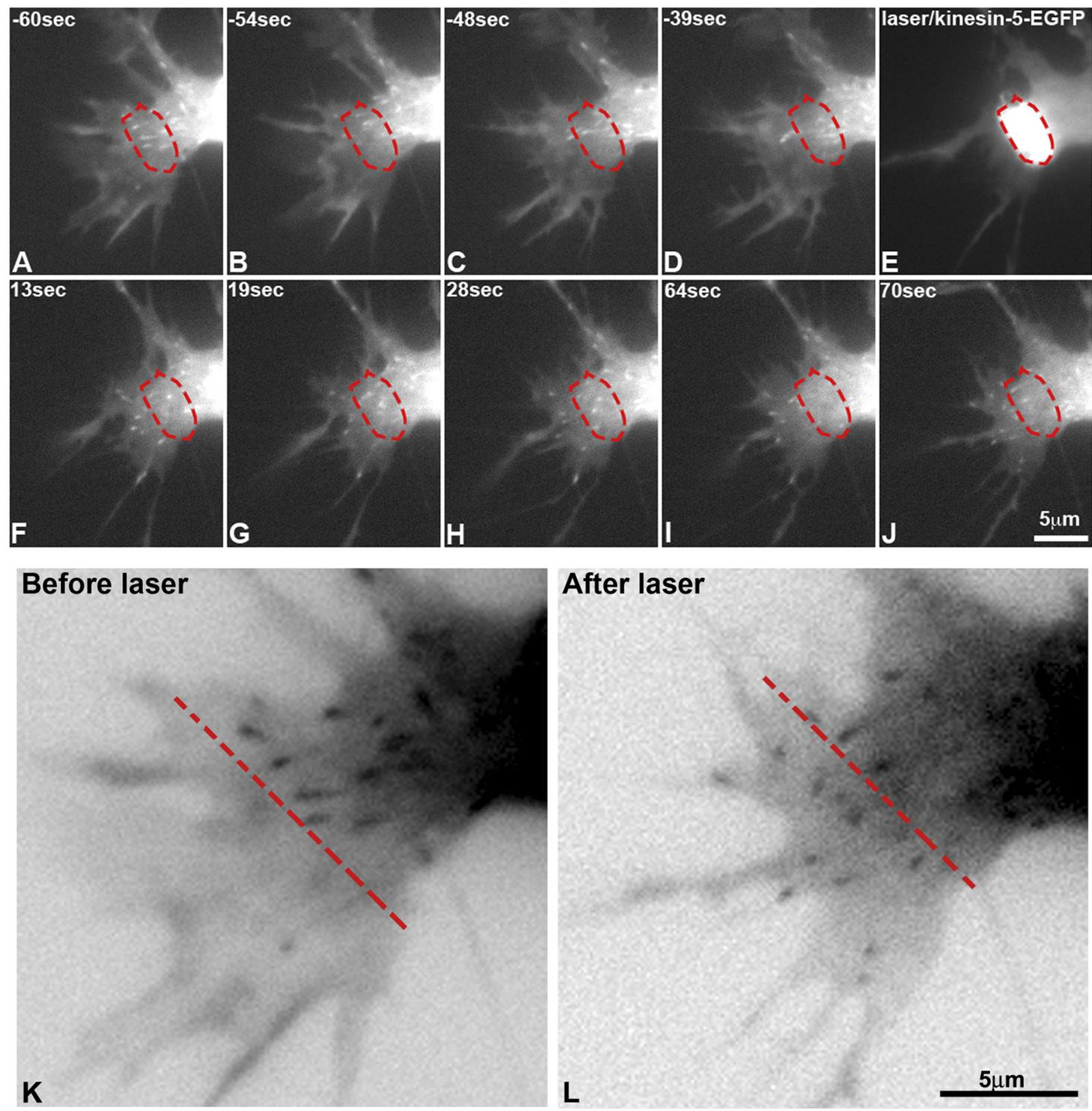

Figure 5. Time-lapse images of a growth cone of a neuron expressing kinesin-5-EGFP and irradiated in the transition zone. $\boldsymbol{A}-\boldsymbol{D}$, Fluorescence time-lapse images of the growth cone before irradiation; the red outline indicates the region of irradiation. Time in negative seconds refers to the time before laser irradiation. $\boldsymbol{E}$, A single fluorescence frame of the same growth cone during laser irradiation. $\boldsymbol{F}$-J, Fluorescence time-lapse images of the growth cone after laser irradiation where the time in seconds refers to the time after laser irradiation. There is a notable increase in microtubule invasion into the peripheral domain as a result of inactivation of kinesin- 5 in the transition zone. Scale bar, $5 \mu \mathrm{m} . \boldsymbol{K}, \boldsymbol{L}$, Enlarged and inverted images of the frames shown in $A$ and $\boldsymbol{J}$.

2002). In these studies, a gradient of the drug was produced by its gradual release from a micropipette using a picospritzer. Frog motor neurons were used because they can be cultured at room temperature, which permits less dissipation of the gradient than with the warmer culture medium required for rodent or avian cultures. If kinesin-5's impact on microtubule distribution as indicated by the micro-CALI studies can cause growth cones to turn, we would expect growth cones to turn toward a gradient of a drug that inhibits kinesin-5. Our previous studies demonstrated that growth cones have an impaired capacity to turn in response to environmental cues if kinesin- 5 is globally inhibited (Nadar et al., 2008), but these studies did not investigate whether local inhibition of kinesin-5 on one side of the growth cone can cause a growth cone to turn. Here, we used the picospritzer approach with frog motor neurons to establish a gradient of monas- trol, an allosteric inhibitor of kinesin-5, which is the same drug we previously bath applied to neurons (Haque et al., 2004; Myers and Baas, 2007; Nadar et al., 2008). The growth cone's response was recorded over a period of $30 \mathrm{~min}$. For control experiments we used DMSO, the vehicle for the monastrol.

We found that a gradient of monastrol induced attractive turning responses toward the monastrol source, whereas the control group did not show any preferential response to a gradient of DMSO. A typical turning of a growth cone toward monastrol is show in Figure $8 A$ and $B$. The average turning angle (in degrees) in the control group was $-0.2 \pm 2.2 \mathrm{SEM}$ and in the monastrol group was $10.1 \pm$ 3.8 SEM ( $p<0.05$, Mann-Whitney $t$ test) (Fig. $8 C$ ). The overall responses of a population of growth cones that showed any turn is depicted in a cumulative histogram of the distribution of the turning angles (Fig. $8 D$ ). Interestingly, the growth cones sometimes bifur- 


\section{EGFP (C-domain)}

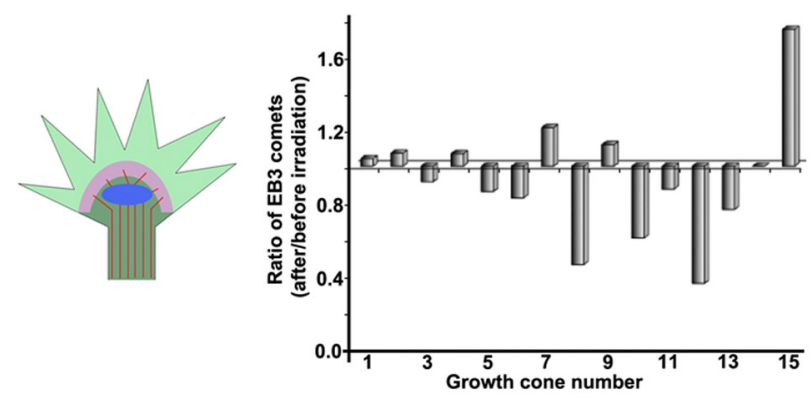

EGFP (P-domain)
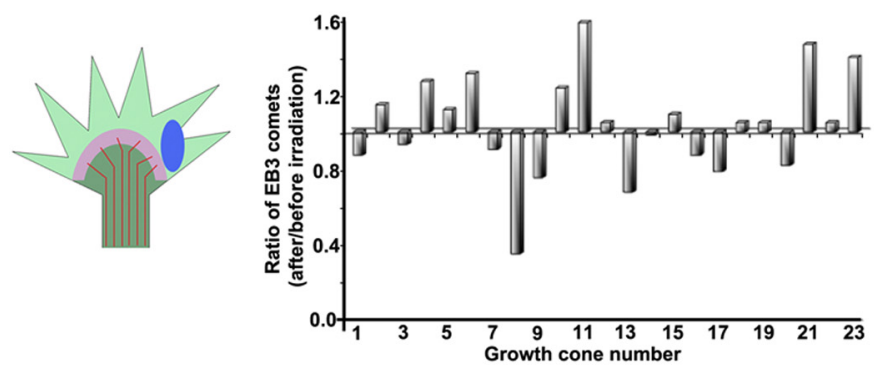

\section{EGFP (T-zone)}
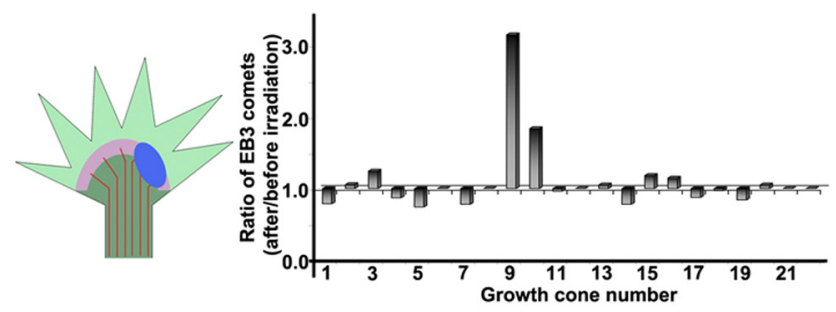

\section{Kinesin-5-EGFP (C-domain)}

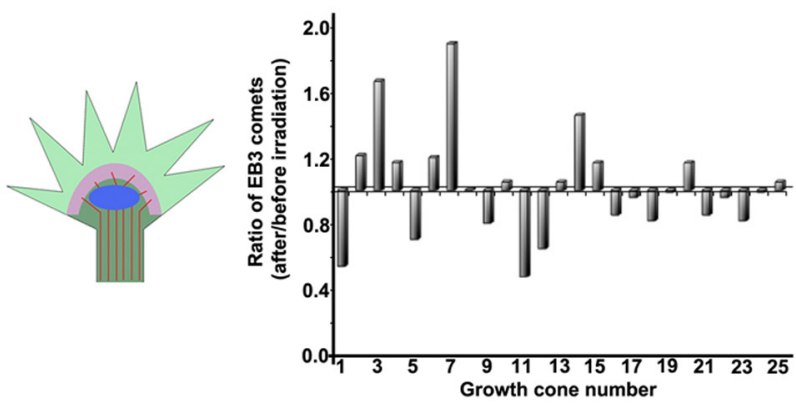

\section{Kinesin-5-EGFP (P-domain)}

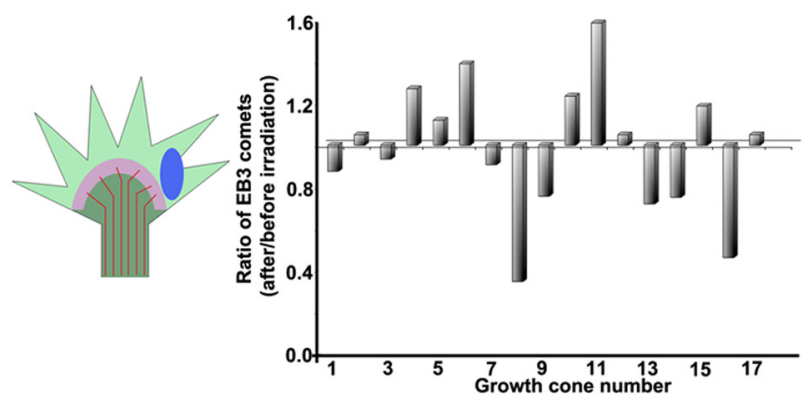

\section{Kinesin-5-EGFP (T-zone)}

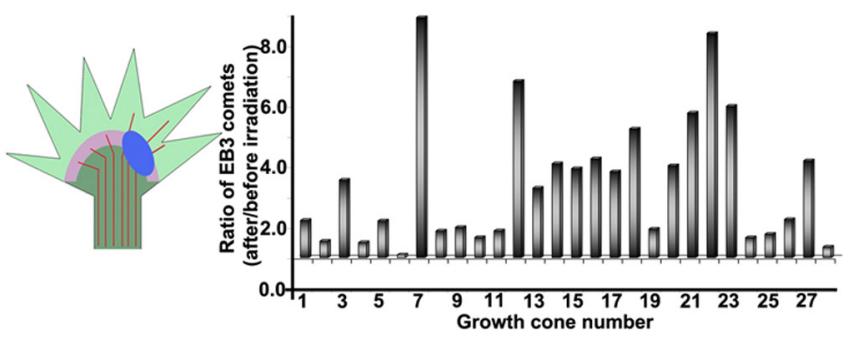

Figure 6. Quantification of EB3 comets invading the peripheral domain of growth cones of neurons expressing either kinesin-5-EGFP or EGFP and irradiated in different regions. The graphs indicate the ratio of EB3 comets invading the peripheral domain after and before laser irradiation on the $y$-axis for each of the growth cones ( $x$-axis) that was irradiated in the central domain, peripheral domain, or transition zone with the adjacent schematic denoting the region of irradiation. Any value $>1$ or a bar above the $x$-axis indicates a positive ratio or an increase in the number of EB3 comets after laser irradiation and vice versa. Consistent with the predictions of our hypothesis (Fig. $3 \mathrm{~A}$ ), there was only a notable increase in microtubule invasion into the peripheral domain when kinesin-5 was inactivated in the transition zone.

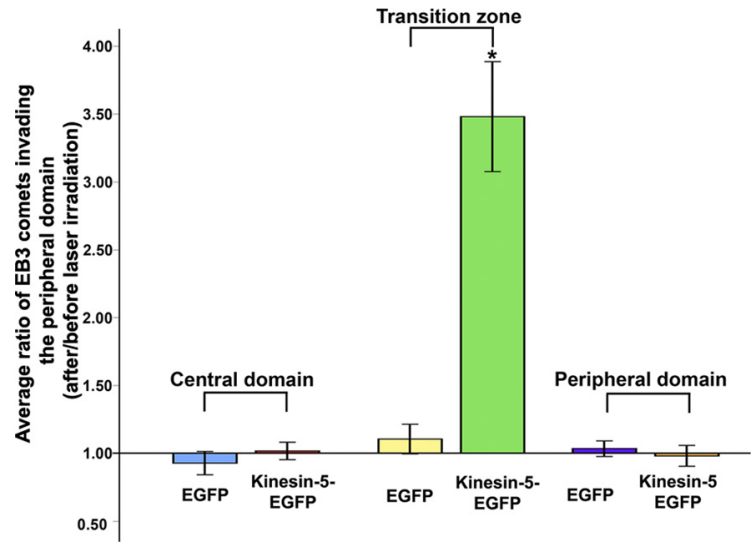

Figure 7. Kinesin-5 functions in the transition zone of the growth cone. Graph showing the average of the ratio of $E B 3$ comets invading the peripheral domain after and before laser irradiation. The data are represented as mean \pm SEM. Consistent with the predictions of our hypothesis (Fig. $3 A$ ), there was only a notable increase in microtubule invasion into the peripheral domain when kinesin-5 was inactivated in the transition zone. cated, with disproportionate growth of the branchlet on the side of the monastrol gradient (Fig. $8 E-H$ ), and sometimes formed a bud on the side of the gradient (Fig. $8 I-K$ ). In cases of growth cones that did not show a turn (where the axon did not tilt toward either side), we determined the ratio of half the growth cone area on the side of the gradient and half growth cone area on the other side. We saw a significant increase in the ratio in growth cone area exposed to the monastrol gradient $(1.7 \pm 0.1, n=15)$ compared with the growth cones exposed to DMSO $(1.1 \pm 0.2, n=9)(p<0.05$, MannWhitney $t$ test) (Fig. $8 L$ ).

Based on the results of the micro-CALI studies, it was our prediction that the growth cones should turn toward a gradient of monastrol, as a result of asymmetric inhibition of kinesin- 5 on the side of the growth cone facing the gradient. Indeed this was the case, but the behaviors were more complex than observed with previous studies using drugs that modify microtubule dynamics. The complex effects on growth cones that we observed suggest that regulation of kinesin-5 may contribute to higher level modifications of growth cone shape and behavior, such as the tendency of the axonal tip to give rise to new branches 

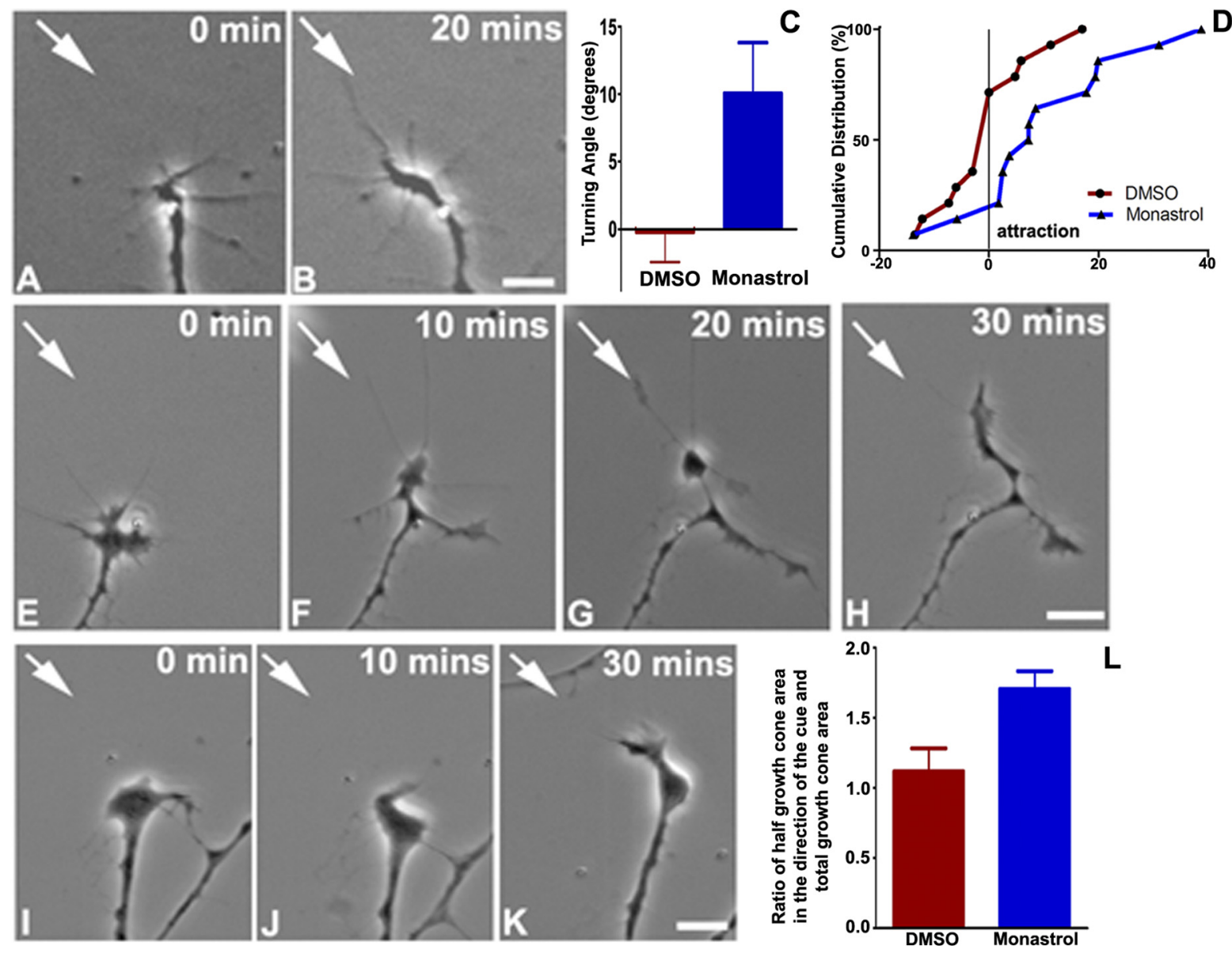

Figure 8. Response of Xenopus neuronal growth cones to local gradient of monastrol. $A, B$, Time-lapse images of Xenopus neuron in which the growth cone tilts toward a gradient of monastrol (the tip of the pipette is indicated by the white arrow). C, Quantification of the average turning angles of the growth cones in response to DMSO (red) and monastrol (blue). D, Frequency distribution of the turning angles for DMSO (red) and monastrol (blue). $\boldsymbol{E}-\boldsymbol{H}$, Time-lapse images of Xenopus neuron in which the growth cone splits in response to the monastrol gradient (the tip of the pipette is indicated by the white arrow). $\boldsymbol{I}-\boldsymbol{K}$, Time-lapse images of Xenopus neuron in which the growth cone forms a bud in response to a gradient of monastrol (the tip of the pipette is indicated by the white arrow). L, Quantification of the ratio of the growth cone area (in the case of growth cones that did not turn, tilt, bud, or branch) on the side toward DMSO or monastrol and growth cone area on the opposite side. Scale bars, $5 \mu \mathrm{m}$.

through bifurcation of the growth cone. Consistent with this conclusion, we previously reported that axons become more highly branched when neurons are depleted of kinesin-5 (Myers and Baas, 2007).

\section{Role of phosphorylation of kinesin-5 in growth cone turning} In our previous study (Nadar et al., 2008), we immunostained neurons with two kinesin-5 antibodies, one which recognizes total kinesin- 5 and one which recognizes kinesin- 5 only when it is phosphorylated at a site within the bimC box of the tail domain. Phosphorylation at this site (Fig. 9A) has been shown in previous studies to be required for kinesin-5 to localize to the mitotic spindle (Blangy et al., 1995; Sawin and Mitchison, 1995). This is presumably because phosphorylation at this site is required for kinesin- 5 to bind to microtubules (Cahu et al., 2008). Our immunofluorescence analyses revealed an enrichment of total kinesin- 5 throughout the transition zone but a more polarized enrichment of phospho-kinesin-5 within the transition zone on the side opposite to the turn. These observations suggested that phosphorylation at this site may be the means by which physiological cues induce kinesin-5 to either engage or disengage microtubules, to elicit its effects. To test this idea, we have now determined the impact on neurons of expressing a nonphosphor- ylatable mutant rat kinesin-5 in which we changed the threonine in the tail domain to alanine (T926A kinesin-5-EGFP). This approach has been used in the past to study the function of this phosphorylation site in dividing cells (Blangy et al., 1995; Sawin and Mitchison, 1995).

We initially tested our T926A kinesin-5-EGFP phosphomutant construct in rat fibroblasts. The analysis of the fixed and immunostained cells undergoing mitosis that had been transfected with either wild-type kinesin-5-EGFP or T926A kinesin-5EGFP confirmed that the former localized to the mitotic spindle (Fig. 9B, $B^{\prime}, B^{\prime \prime}$ ), while the latter did not (Fig. 9C, $C^{\prime}, C^{\prime \prime}$ ). Somewhat surprisingly, during mitosis, the phospho-mutant does not display obvious dominant-negative effects, perhaps because virtually all of the available kinesin- 5 molecules are concentrated on spindle microtubules. With regard to neurons, our hypothesis is that there is a delicate balance between phosphorylated and nonphosphorylated sites, and hence our expectation was that a relatively mild expression of the phospho-mutant should have profound phenotypic effects. As a first test of this, we analyzed the effect of expressing the T926A kinesin-5-EGFP construct on the length of the axon. We expressed wild-type kinesin-5-EGFP or T926A kinesin-5-EGFP in neurons and observed that the axons of neurons transfected with the latter (Fig. $9 D, D^{\prime}, D^{\prime \prime}$ ) were longer 
A

\section{STRUCTURE OF KINESIN-5 MOLECULE}

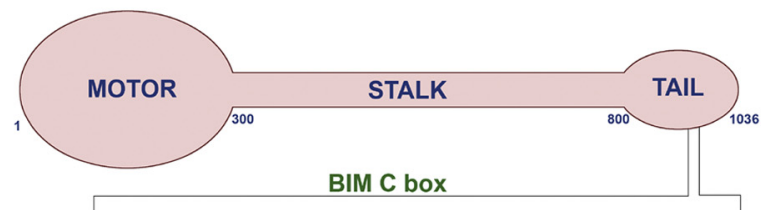

DLKLDIPTGMTPERKNYLYPSTLVRTEPREQLLDQLQKKQ Cdk consensus site S/TPxK/R
Tubulin
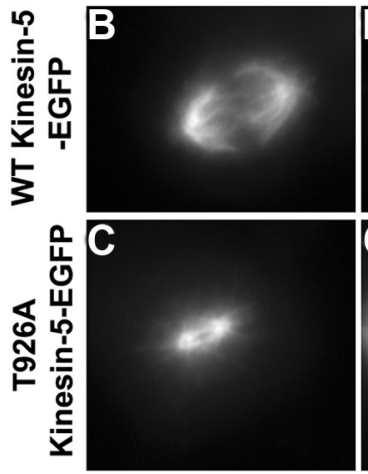

EGFP
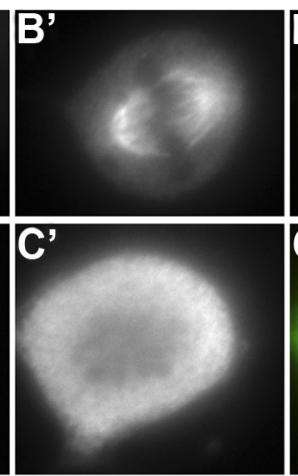

Overlay

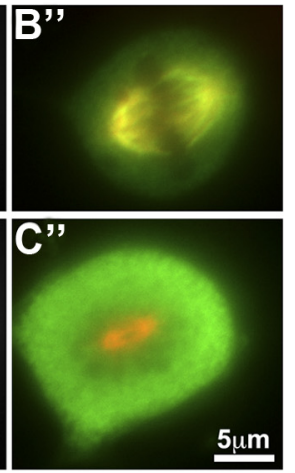

EGFP
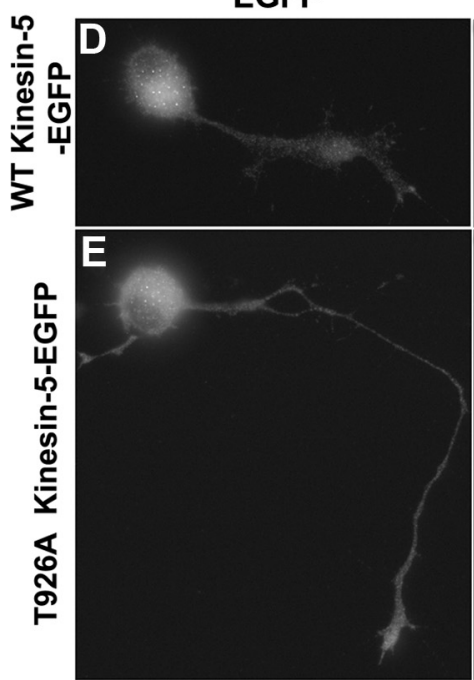

Tubulin
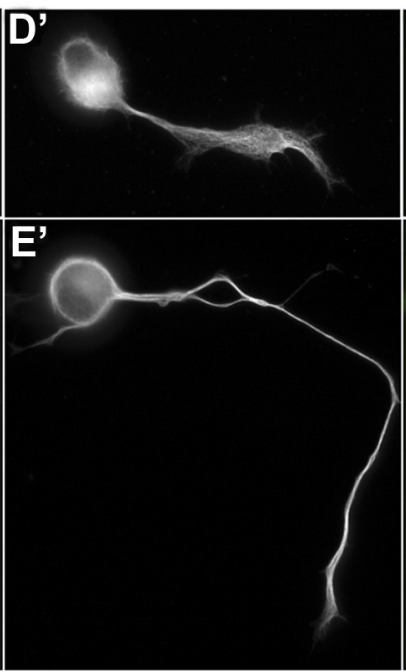
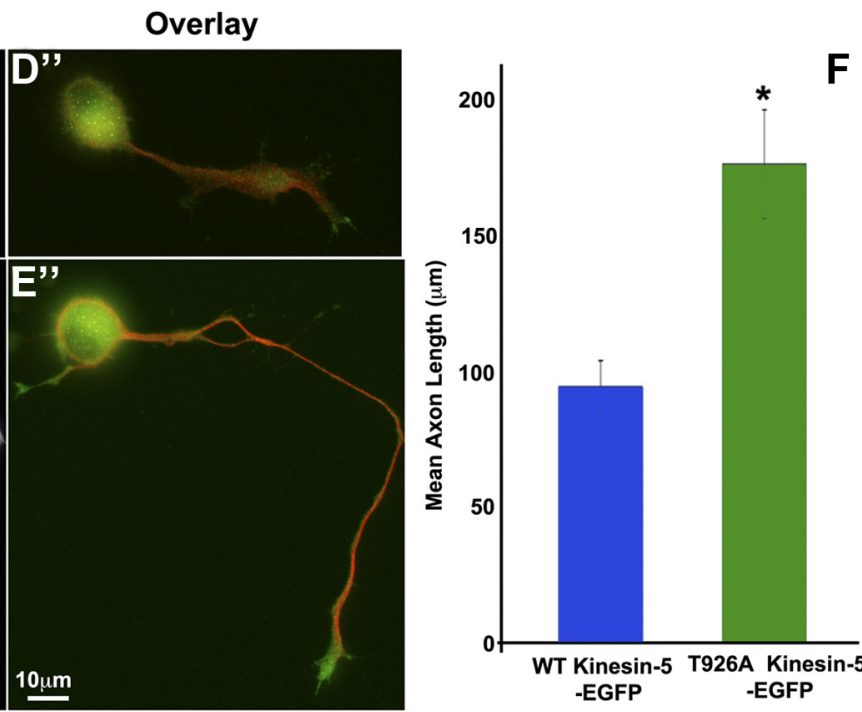

Figure 9. Effect of expression of T926A kinesin-5-EGFP on mitotic cells and axonal length. $A$, Schematic of the structure of the kinesin-5 molecule. The tail domain contains the bim $C$ box that has a conserved cdk consensus site. $\boldsymbol{B}^{\prime} \boldsymbol{B}^{\prime}, \boldsymbol{B}^{\prime \prime}, \boldsymbol{C}, \boldsymbol{C}^{\prime}, \boldsymbol{C}^{\prime}$, Fluorescence images of RFL-6 cells transfected to express GFP fusions for wild-type kinesin-5 $\left(\boldsymbol{B}^{\prime}, \boldsymbol{B}^{\prime}, \boldsymbol{B}^{\prime \prime}\right)$ or mutant T926A kinesin-5 $\left(\boldsymbol{C}, \boldsymbol{C}^{\prime}, \boldsymbol{C}^{\prime \prime}\right)$. $\boldsymbol{B}$, C, Tubulin immunostains. $\boldsymbol{B}^{\prime}, \boldsymbol{C}^{\prime}$ Immunostains with GFP antibody to accentuate EGFP fluorescence. $\boldsymbol{B}^{\prime \prime}, \boldsymbol{C}^{\prime \prime}$, Overlap of tubulin (yellow) and GFP (green) images. Note that wild-type kinesin-5 associates with the mitotic spindle microtubules, whereas the mutant does not. Scale bar, $5 \mu \mathrm{m} . \boldsymbol{D}, \boldsymbol{D}^{\prime}, \boldsymbol{D}^{\prime \prime}, \boldsymbol{E}, \boldsymbol{E}^{\prime}, \boldsymbol{E}^{\prime \prime}$, Fluorescence images of neurons transfected to express GFP fusions for wild-type kinesin-5 $\left(\boldsymbol{D}, \boldsymbol{D}^{\prime}, \boldsymbol{D}^{\prime \prime}\right)$ or mutant T926A kinesin-5 $\left(\boldsymbol{E}, \boldsymbol{E}^{\prime}, \boldsymbol{E}^{\prime \prime}\right) . \boldsymbol{D}, \boldsymbol{E}$, Immunostains with GFP antibody to accentuate EGFP fluorescence. $\boldsymbol{D}^{\prime}, \boldsymbol{E}^{\prime}$, Tubulin immunostains. $\boldsymbol{B}^{\prime \prime}, \boldsymbol{C}^{\prime \prime}$, Overlap of tubulin (red) and GFP (green) images. Neurons transfected with the mutant display a notably longer axon than neurons transfected with wild-type kinesin-5, consistent with a dominant-negative effect of the mutant. (Axons of neurons transfected with wild-type kinesin-5 were sometimes slightly shorter than control neurons, consistent with an overexpression phenotype, but this was not statistically significant for moderate expressers). Scale bar, $10 \mu \mathrm{m}$. $\boldsymbol{F}$, Quantification of axonal length. ${ }^{*} p \leq 0.001$.

than the axons of neurons transfected with the former (Fig. $\left.9 E, E^{\prime}, E^{\prime \prime}\right)$. For these analyses, we selected neurons with similar expression levels, as determined by fluorescence intensity. We chose moderate expressers, because their axonal lengths in the case of the wild-type were within the same range as the nonexpressing neurons. Higher levels of expression can result in an overexpression phenotype of notably shorter axons (Myers and Baas, 2007). Quantification revealed a significant increase in axonal lengths of neurons expressing the T926A kinesin-5-EGFP $(177.3 \pm 20.0)(n=37)$ compared with axonal lengths of neurons expressing the wild-type kinesin-5-EGFP $(94.3 \pm 9.7)(n=$ 32) ( $p \leq 0.001$ two-tailed $t$ test) (Fig. $9 F$ ). Notably, the impact on axonal length of expressing T926A kinesin-5-EGFP is almost identical to the impact of depleting kinesin-5 with siRNA (Myers and Baas, 2007), consistent with the phospho-mutant acting as a dominant-negative when expressed in neurons.

Next, we tested whether expression of T926A kinesin-5-EGFP impacts the distribution of microtubules in growth cones. Previously, we showed that global depletion of kinesin-5 (or treatment with the kinesin-5 inhibitor monastrol) results in increased mi- crotubule invasion throughout the peripheral domain (Nadar et al., 2008). Neurons were cotransfected with wild-type kinesin-5EGFP or T926A kinesin-5-EGFP and with mCherry-EB3 to visualize microtubule behaviors. Live-cell imaging of the EB3 comets revealed an increase in the number of EB3 comets in the peripheral domain in the growth cones of neurons transfected with T926A kinesin-5-EGFP and fewer comets in the peripheral domain of the growth cones of neurons transfected with wild-type kinesin-5-EGFP (Fig. 10A,B). Quantification of the number of EB3 comets in the peripheral domain normalized over the growth cone area showed a significant increase in growth cones of T926A-transfected neurons $(0.01 \pm 0.0009)(n=20)$ compared with growth cones of neurons transfected with wild-type kinesin5 -EGFP $(0.005 \pm 0.0006)(n=20) ; p \leq 0.00001$ (two-tailed $t$ test) (Fig. 10C). Similar to the impact on axonal length, these results indicate that the T926A mutant produces almost identical results to global depletion of kinesin-5 by siRNA or pharmacologic inhibition of kinesin-5 by monastrol. In contrast, the expression of the wild-type kinesin-5 at these levels had only modest indications of an overexpression phenotype. Collectively, 

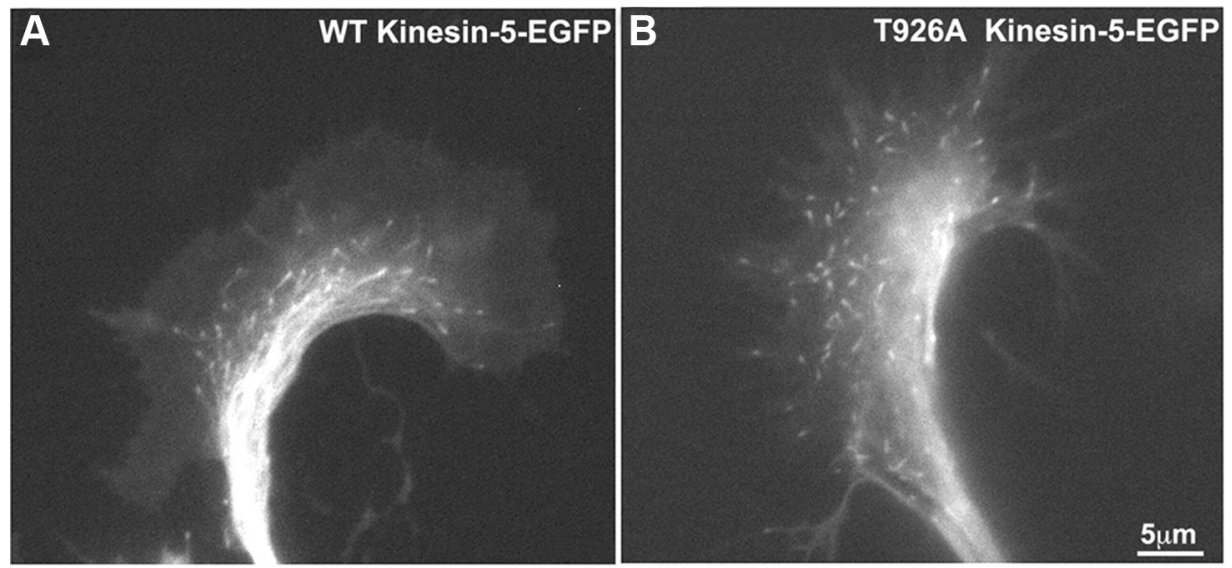

WT Kinesin-5-EGFP

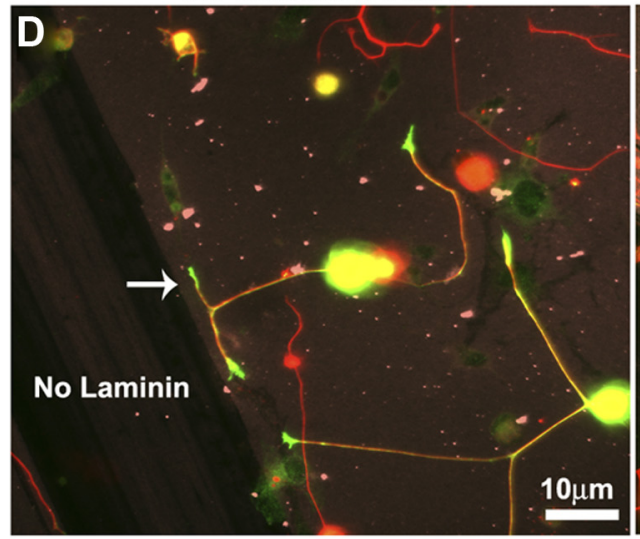

T926A Kinesin-5-EGFP

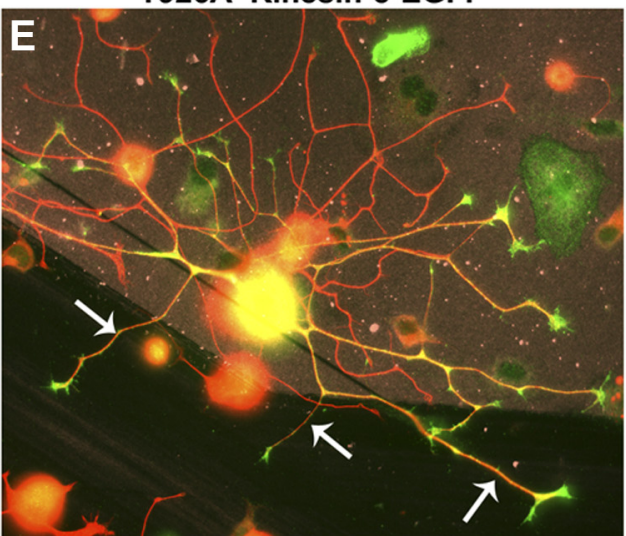

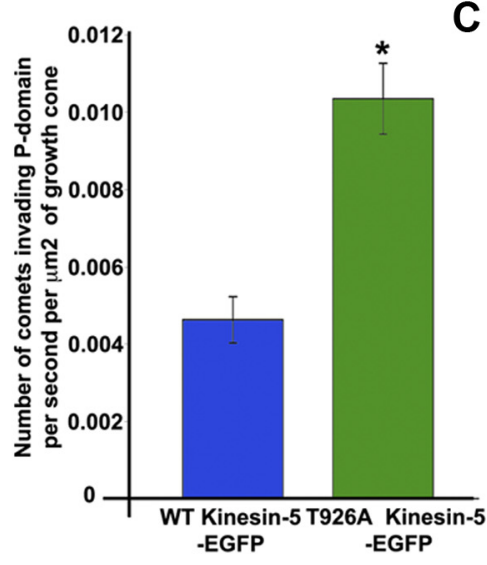

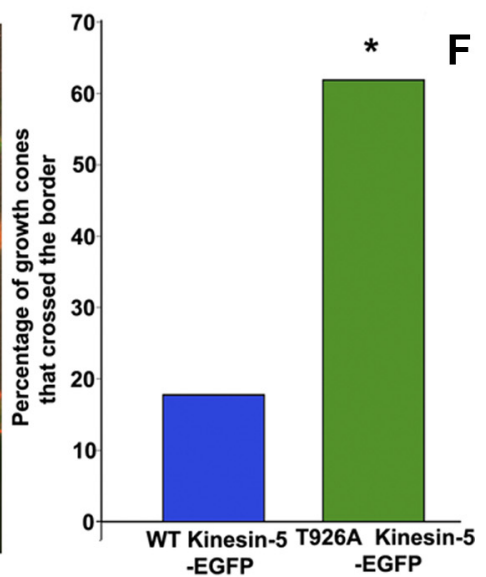

Figure 10. Effect of expression of T926A kinesin-5-EGFP on growth cone behavior. $\boldsymbol{A}, \boldsymbol{B}$, Fluorescence images of growth cones of neurons expressing mCherry-EB3 and either wild-type kinesin-5-EGFP or T926A kinesin-5-EGFP, respectively. Notably more EB3 comets invade the peripheral domain in neurons transfected with the mutant, consistent with a dominant-negative effect. Scale bar, $5 \mu \mathrm{m}$. C, Quantification of the number of EB3 comets that enter the peripheral domain. ${ }^{*} p \leq 0.00001 . D, E$, Fluorescence images of neurons expressing wild-type kinesin-5-EGFP or T926A kinesin-5-EGFP, respectively, that were plated on border preparations of laminin and nonlaminin areas. Tubulin (red), green (GFP), laminin (orange). White arrow in $\boldsymbol{D}$ indicates an axon that did not cross the border and in $\boldsymbol{E}$ indicates axons that crossed the border. Notably more axons cross the border in the case of neurons expressing the mutant, consistent with a dominant-negative effect of the mutant. Axons of neurons expressing the wild-type construct were generally shorter than axons of neurons expressing the mutant construct. Scale bar, $10 \mu \mathrm{m}$. $\boldsymbol{F}$, Quantification of the percentage of axons that crossed the border. ${ }^{*} p \leq 0.0001$.

these observations provide confidence that the phospho-mutant acts as a dominant-negative, which is consistent with the need for kinesin-5 to interact with microtubules to elicit its functions.

Depletion or inhibition of kinesin-5 in neurons inhibits growth cone turning (Nadar et al., 2008), and therefore in a final set of studies, we evaluated the effects of the T926A mutant on the ability of growth cones to turn at a laminin/nonlaminin border. For this, we used a scratch/border assay in which an axon was scored as having turned if the angle between the long axis of the axon and the nonlaminin region was $<45^{\circ}$ (Myers et al., 2006). The growth cones of neurons expressing wild-type kinesin-5EGFP did not cross the border but stayed on laminin, similar to the growth cones of untransfected neurons (Fig. 10D). In contrast, growth cones of neurons expressing the T926A kinesin-5EGFP crossed the border and continued to grow on the nonlaminin surface (Fig. 9E). Quantification of the turning behavior of transfected growth cones that reached the border revealed that a significantly higher number of growth cones crossed the border in neurons transfected with T926A kinesin-5-EGFP $(62 \%)$ compared with growth cones of neurons expressing wildtype kinesin-5-EGFP $(18 \%)\left(p<0.0001, \chi^{2}\right.$ test $)$ (Fig. 10F). This suggests that phosphorylation of kinesin- 5 in the tail domain is critical for it to function properly in growth cone turning.

\section{Discussion}

There is broad agreement that in order for a growth cone to turn toward an environmental cue, microtubules must be preferentially recruited into filopodia on the side of the growth cone facing the direction of the turn (Williamson et al., 1996; Mack et al., 2000). Studies to date have documented that the dynamic properties of the microtubules are essential for growth cone turning (Challacombe et al., 1997; Buck and Zheng, 2002; Geraldo and Gordon-Weeks, 2009), presumably because long microtubules from the central domain must assemble into the relevant filopodia. However, it remains unclear how individual microtubules are able to overcome the retrograde flow of the actin cytoskeleton in a specific region of the growth cone to penetrate from the central domain through the transition zone to enter the peripheral domain and ultimately invade filopodia. The regulatory mechanisms that control this process must be responsive to signaling events so that the invasion of microtubules can be polarized. Our studies over the past several years have taken inspiration from the mitotic spindle (Baas, 1999), where the dynamic properties of the microtubules are critical, but so too are the forces generated on the microtubules by molecular motor proteins (Kollu et al., 2009). We posit that the same is true of the 
growth cone, where we speculate that the distribution of microtubules is regulated by both their dynamic properties and by forces generated on them by molecular motors. In fact, our work on growth cones implicates some of the very same motors that regulate microtubules in the mitotic spindle.

Within the peripheral domain of the growth cone, there is a powerful retrograde flow of the actin cytoskeleton. This flow, which is driven by myosin-II, pushes the microtubules back toward the axonal shaft, compacting them in the central domain of the growth cone (Burnette et al., 2008). Forces generated on the microtubule array by cytoplasmic dynein are able to partially counterbalance the retrograde actin flow, enabling a portion of the microtubules from the central domain to enter the peripheral domain (Myers et al., 2006; Grabham et al., 2007). In theory, a growth cone could turn by spatial regulation of the forces generated by either cytoplasmic dynein or myosin-II, but thus far, there is no compelling evidence that this is how the microtubule array is polarized to elicit growth cone turning. Our premise is that it is the contribution of other motors, namely the mitotic motors, that tips the balance of forces in a fashion that polarizes the microtubule array and hence permits the growth cone to turn. This is an appealing idea because the mitotic motors are known to be exquisitely sensitive to signaling cascades.

Over a decade ago we documented that kinesin- 5 is robustly expressed in developing neurons and is concentrated in growth cones (Ferhat et al., 1998). Our more recent studies demonstrated that global inhibition of kinesin-5 with monastrol or depletion of kinesin- 5 with siRNA results in a faster growing axon (Haque et al., 2004; Myers and Baas, 2007) with a broader growth cone (Liu et al., 2010) that does not turn properly in response to environmental cues (Nadar et al., 2008). The lack of growth cone turning was explicable on the basis of a widespread increase in microtubule invasion throughout the peripheral domain when kinesin-5 was inhibited or depleted. On this basis, we speculated that kinesin-5 normally elicits its forces disproportionately on the side of the growth cone opposite the direction of turning, thus suppressing microtubule invasion on that side of the cone compared with the other side. Support for this idea came from immunofluorescence analyses showing enrichment of kinesin-5 in the transition zone, with more microtubules invading the peripheral domain in regions in which phosphorylated kinesin-5 was less abundant in the corresponding region of the transition zone (Nadar et al., 2008). Phosphorylation of kinesin-5 within its bimC box is important for its ability to associate with microtubules (Cahu et al., 2008), and hence makes sense as a potential means by which kinesin-5's activity can be regulated by signaling cascades. In support of this conclusion, studies presented here indicate that a phospho-mutant corresponding to this site behaves as a dominant-negative when expressed in neurons.

Given the complex organization of the growth cone cytoskeleton, knowing where kinesin-5's forces are most relevant is critical for understanding the mechanism by which it elicits its effects. Live-cell imaging of fluorescently tagged kinesin-5 revealed the presence of kinesin-5 in all regions of the growth cone, with enrichment in the transition zone, consistent with our previous immunofluorescence results. In the peripheral domain, kinesin-5 sometimes appeared as streak-like blurs that roughly/ partially colocalize with the invading microtubules. We speculate that kinesin-5 interacts with neighboring microtubules in the transition zone (in a manner that is functionally important) but then "dashes" forward along individual microtubules as they extend into the peripheral domain. However, on the basis of these observations alone, we could not dismiss the peripheral domain or any region of the growth cone as a potential site for kinesin-5 to elicit its functional effects. To resolve this, we used micro-CALI to focally inactivate kinesin-5 in various regions of the growth cone and then observed the effects on microtubule distribution. Consistent with our original hypothesis, we found that focally inactivating kinesin-5 in the transition zone resulted in robust invasion of microtubules into the corresponding region of the peripheral domain. No such effects were observed when kinesin- 5 was focally inactivated in the peripheral domain itself or in the central domain. Thus, we would envision a scenario in which the transition zone is where a tug-of-war occurs between the forces of kinesin-5 and cytoplasmic dynein. In particular regions of the transition zone, kinesin-5 "loses the war" and microtubules are able to extend forward into the peripheral domain. If this happens, kinesin- 5 dashes along individual microtubules as they splay apart from their neighbors, ultimately to fall off at the microtubule's plus end. The kinesin- 5 molecules can then be recycled and used again in the transition zone.

Kinesin-5 is unique among kinesins in that it forms homotetramers, with four motor domains projected outward (Kashina et al., 1997). Thus, it has no traditional cargo domain, and can only move along microtubules or generate forces between neighboring microtubules. Kinesin-5 is specialized to drive apart microtubules of opposite orientation and appears to preferentially interact with anti-parallel regions of microtubule arrays (van den Wildenberg et al., 2008). Because it is a very slow motor, kinesin-5 can function as a "brake" to slow the rate at which the microtubules would be moved apart by other molecular motors (Saunders et al., 2007). In the axon, nearly all the microtubules have the same orientation (Baas and Lin, 2011), which prompts the question of how kinesin-5 might function in growth cones. The transition zone makes sense as the site of action for kinesin-5 because the microtubules are packed together by the retrograde actin flow, with many of the microtubules folding back on themselves as well as their neighbors, thus creating potential areas of anti-parallel overlap. Such regions would be ideal sites for kinesin-5 to impose a brake on the dynein-driven forces that would otherwise drive the microtubules into the peripheral domain. Such a scenario is consistent with previous studies, suggesting that kinesin-5 and cytoplasmic dynein often antagonize one another (Gaglio et al., 1996; Ferenz et al., 2009).

Unfortunately the micro-CALI approach does not permit focal inactivation over a period of time that would be required for the polarization of the microtubule array to produce a turning event. However, we found that growth cones turn toward a gradient of monastrol (a potent anti-kinesin-5 drug), which is what we would have predicted on the basis of the micro-CALI results. Interestingly, the response to monastrol was more complex than the response to drugs that impact microtubule dynamics as sometimes the growth cone generated a bud or branchlet in the direction of the monastrol gradient, rather than just tilting toward it. We suspect that this is because monastrol can result in an en masse entry of many microtubules, while growth cone turning requires the invasion of only an exceedingly small number of microtubules. These results, and our earlier results indicating enhanced branching of axons of sympathetic neurons depleted of kinesin-5 (Myers and Baas, 2007), suggest that kinesin-5 might play an important role in axonal branching via growth cone bifurcation.

With regard to kinesin-5 regulation, phosphorylation in the bimC box is the most obvious possibility. However, kinesin- 5 has other sites that can be phosphorylated (Rapley et al., 2008) as well as other sites that are important for its interactions with micro- 
tubules (Weinger et al., 2011). There are also regulatory proteins that interact with kinesin-5 such as TPX2 (Ma et al., 2010), and at least one other motor protein, namely kinesin-12, with functions that partially overlap with those of kinesin-5 (Tanenbaum et al., 2008; Liu et al., 2010). The next important step will be to ascertain how these various factors coordinate with one another under the influence of signaling cascades to orchestrate the behaviors of the growth cone.

\section{References}

Aletta JM, Greene LA (1988) Growth cone configuration and advance: a time-lapse study using video enhanced differential interference contrast microscopy. J Neurosci 8:1425-1435.

Baas PW (1999) Microtubules and neuronal polarity: lessons from mitosis. Neuron 22:23-31.

Baas PW, Lin S (2011) Hooks and comets: the story of microtubule polarity orientation in the neuron. Dev Neurobiol 71:403-418.

Blangy A, Lane HA, d'Herin P, Harper M, Kress M, Nigg EA (1995) Phosphorylation by p34cdc2 regulates spindle association of human Eg5, a kinesin-related motor essential for bipolar spindle formation in vivo. Cell 83:1159-1169.

Buck KB, Zheng JQ (2002) Growth cone turning induced by direct local modification of microtubule dynamics. J Neurosci 22:9358-9367.

Burnette DT, Ji L, Schaefer AW, Medeiros NA, Danuser G, Forscher P (2008) Myosin II activity facilitates microtubule bundling in the neuronal growth cone neck. Dev Cell 15:163-169.

Cahu J, Olichon A, Hentrich C, Schek H, Drinjakovic J, Zhang C, DohertyKirby A, Lajoie G, Surrey T (2008) Phosphorylation by Cdk1 increases the binding of Eg5 to microtubules in vitro and in Xenopus egg extract spindles. PLoS One 3:e3936.

Challacombe JF, Snow DM, Letourneau PC (1997) Dynamic microtubule ends are required for growth cone turning to avoid an inhibitory guidance cue. J Neurosci 17:3085-3095.

Cole DG, Saxton WM, Sheehan KB, Scholey JM (1994) A "slow" homotetrameric kinesin related motor protein purified from Drosophila embryos. J Biol Chem 269:22913-22916.

Conde C, Caceres A (2009) Microtubule assembly, organization and dynamics in axons and dendrites. Nat Rev Neurosci 10:319-332.

Dent EW, Gupton SL, Gertler FB (2011) The growth cone cytoskeleton in axon outgrowth and guidance. Cold Spring Harb Perspect Biol 3:pii: a001800.

Ferenz NP, Paul R, Fagerstrom C, Mogilner A, Wadsworth P (2009) Dynein antagonizes Eg5 by crosslinking and sliding antiparallel microtubules. Curr Biol 19:1833-1838.

Ferhat L, Cook C, Chauviere M, Harper M, Kress M, Lyons GE, Baas PW (1998) Expression of the mitotic motor protein Eg5 in postmitotic neurons: implications for neuronal development. J Neurosci 18:7822-7835.

Gaglio T, Saredi A, Bingham JB, Hasbani MJ, Gill SR, Schroer TA, Compton DA (1996) Opposing motor activities are required for the organization of the mammalian mitotic spindle pole. J Cell Biol 135:399-414.

Geraldo S, Gordon-Weeks PR (2009) Cytoskeletal dynamics in growthcone steering. J Cell Sci 122:3595-3604.

Goldberg DJ, Burmeister DW (1986) Stages in axon formation: observations of growth of Aplysia axons in culture using video-enhanced contrastdifferential interference contrast microscopy. J Cell Biol 103:1921-1931.

Gomez TM, Robles E (2003) Imaging calcium dynamics in developing neurons. Methods Enzymol 361:407-422.

Grabham PW, Seale GE, Bennecib M, Goldberg DJ, Vallee RB (2007) Cytoplasmic dynein and LIS1 are required for microtubule advance during growth cone remodeling and fast axonal outgrowth. J Neurosci 27:5823-5834.

Guirland C, Buck KB, Gibney JA, DiCicco-Bloom E, Zheng JQ (2003) Direct cAMP signaling through G-protein-coupled receptors mediates growth cone attraction induced by pituitary adenylate cyclase-activating polypeptide. J Neurosci 23:2274-2283.

Haque SA, Hasaka TP, Brooks AD, Lobanov PV, Baas PW (2004) Monastrol, a prototype anticancer drug that inhibits a mitotic kinesin, induces rapid bursts of axonal outgrowth from cultured postmitotic neurons. Cell Motil Cytoskeleton 58:10-16.

He Y, Francis F, Myers KA, Yu W, Black MM, Baas PW (2005) Role of cytoplasmic dynein in the axonal transport of microtubules and neurofilaments. J Cell Biol 168:697-703.

Kashina AS, Baskin RJ, Cole DG, Wedaman KP, Saxton WM, Scholey JM (1996) A bipolar kinesin. Nature 379:270-272.

Kashina AS, Rogers GC, Scholey JM (1997) The bimC family of kinesins: essential bipolar mitotic motors driving centrosome separation. Biochim Biophys Acta 1357:257-271.

Koester MP, Muller O, Pollerberg GE (2007) Adenomatous polyposis coli is differentially distributed in growth cones and modulates their steering. J Neurosci 27:12590-12600.

Kollu S, Bakhoum SF, Compton DA (2009) Interplay of microtubule dynamics and sliding during bipolar spindle formation in mammalian cells. Curr Biol 19:2108-2113.

Liu CW, Lee G, Jay DG (1999) Tau is required for neurite outgrowth and growth cone motility of chick sensory neurons. Cell Motil Cytoskeleton 43:232-242.

Liu M, Nadar VC, Kozielski F, Kozlowska M, Yu W, Baas PW (2010) Kinesin-12, a mitotic microtubule-associated motor protein, impacts axonal growth, navigation, and branching. J Neurosci 30:14896-14906.

Lowery LA, Van Vactor D (2009) The trip of the tip: understanding the growth cone machinery. Nat Rev Mol Cell Biol 10:332-343.

Ma N, Tulu US, Ferenz NP, Fagerstrom C, Wilde A, Wadsworth P (2010) Poleward transport of TPX2 in the mammalian mitotic spindle requires dynein, Eg5, and microtubule flux. Mol Biol Cell 21:979-988.

Mack TG, Koester MP, Pollerberg GE (2000) The microtubule-associated protein MAP1B is involved in local stabilization of turning growth cones. Mol Cell Neurosci 15:51-65.

McLean MA, Rajfur Z, Chen Z, Humphrey D, Yang B, Sligar SG, Jacobson K (2009) Mechanism of chromophore assisted laser inactivation employing fluorescent proteins. Anal Chem 81:1755-1761.

Medeiros NA, Burnette DT, Forscher P (2006) Myosin II functions in actinbundle turnover in neuronal growth cones. Nat Cell Biol 8:215-226.

Myers KA, Baas PW (2007) Kinesin-5 regulates the growth of the axon by acting as a brake on its microtubule array. J Cell Biol 178:1081-1091.

Myers KA, He Y, Hasaka TP, Baas PW (2006) Microtubule transport in the axon: re-thinking a potential role for the actin cytoskeleton. Neuroscientist 12:107-118.

Nadar VC, Ketschek A, Myers KA, Gallo G, Baas PW (2008) Kinesin-5 is essential for growth-cone turning. Curr Biol 18:1972-1977.

Rapley J, Nicolas M, Groen A, Regue L, Bertran MT, Caelles C, Avruch J, Roig J (2008) The NIMA-family kinase Nek6 phosphorylates the kinesin Eg5 at a novel site necessary for mitotic spindle formation. J Cell Sci 121:3912-3921.

Saunders AM, Powers J, Strome S, Saxton WM (2007) Kinesin-5 acts as a brake in anaphase spindle elongation. Curr Biol 17:R453-R454.

Sawin KE, LeGuellec K, Philippe M, Mitchison TJ (1992) Mitotic spindle organization by a plus-end-directed microtubule motor. Nature 359:540-543.

Stepanova T, Slemmer J, Hoogenraad CC, Lansbergen G, Dortland B, De Zeeuw CI, Grosveld F, van Cappellen G, Akhmanova A, Galjart N (2003) Visualization of microtubule growth in cultured neurons via the use of EB3-GFP (end-binding protein 3-green fluorescent protein). J Neurosci 23:2655-2664.

Suter DM, Schaefer AW, Forscher P (2004) Microtubule dynamics are necessary for SRC family kinase-dependent growth cone steering. Curr Biol 14:1194-1199.

Tanaka E, Kirschner MW (1995) The role of microtubules in growth cone turning at substrate boundaries. J Cell Biol 128:127-137.

Tanenbaum ME, Macurek L, Galjart N, Medema RH (2008) Dynein, Lis1 and CLIP-170 counteract Eg5-dependent centrosome separation during bipolar spindle assembly. EMBO J 27:3235-3245.

van den Wildenberg SM, Tao L, Kapitein LC, Schmidt CF, Scholey JM, Peterman EJ (2008) The homotetrameric kinesin-5 KLP61F preferentially crosslinks microtubules into antiparallel orientations. Curr Biol 18:1860-1864.

Weinger JS, Qiu M, Yang G, Kapoor TM (2011) A nonmotor microtubule binding site in kinesin-5 is required for filament crosslinking and sliding. Curr Biol 21:154-160.

Williamson T, Gordon-Weeks PR, Schachner M, Taylor J (1996) Microtubule reorganization is obligatory for growth cone turning. Proc Natl Acad Sci U S A 93:15221-15226. 